\title{
N91-31795
}

\section{TRANSPIRATION DURING LIFE CYCLE IN CONTROLLED WHEAT GROWTH}

\author{
Tyler Volk* and John D. Rummel** \\ *Earth Systems Group, Department of Applied Science, \\ New York University. New York, NY 10003, U.S.A. \\ ** Code EBR, Life Sciences, NASA Headquarters. \\ Washington, D.C. 20546, U.S.A.
}

\section{ABSTRACT}

We We apply the model to data from an experiment that system-level models of advanced space life suppor systems. We apply the model to data fremsionation as a function of grew wheat under controlled conditions and measured fresh biomass and curnulated transpiration as a funs and an age time. We examine the adequacy of modeling the transpiration as proportional to the inedible biomass and an age factor, which varies during the life cycle. Results indicate that during the main phase of vegetative gan double the rate first half of the life cycle, the rate of transpiration per unit mass of inedible biomass is

during the phase of grain development and maturation during latter half of the life cycle.

\section{THE GROWTH MODEL}

We developed a model for the growth of wheat /1/, which proved useful for coupling the wheat with other (CELSS). The rationale for the model's form is that growth curves of most crops prominently show the $S$-shaped or sigmoidal curve typical of 列

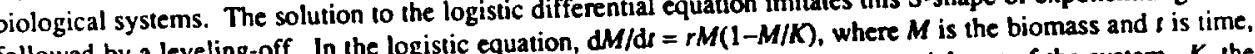
followed by a leveling-off. In the logistic equation, $d M / d r=$ for the purely exponential part of the system. $K$, the there are two parameters: $r$ and $K$. The $r$ is the growth rate for the purely exponential part the crop. The logistic carrying-capacity in an ecological system, in this case is the maxim
equation thus contains some biologically meaningful parameters.

While the logistic equation can be applied directly to the growth of the inedible biomass of a crop, the equation for (he edible cells the edice and the total edible growth must be proportional to the edible mass $\left(M_{\mathrm{Cd}}\right)$. However, the edible parts do ine not produce their growing mass through photosynthesis, but biomass $\left(M_{\text {ined }}\right)$ should also appear in the edible inedible parts (in particular, the leaves); therefore, the inedible bions ( $M$ ined) should also appear in the ef inedible growth equation. Furthermore, since the edible growth occurs substanilites the growth of the edible mass $\left(t^{*}\right)$ is (about halfway through the life cycle for wheat), a time that inits is assumed equal to zero, and its growth is

incorporated into the edible equation. Before $t^{*}$ the edible biomass is assumed equal $n$, 2 :

$$
\begin{aligned}
& \frac{\mathrm{d} M_{\text {inod }}}{\mathrm{d} t}=r_{\text {incd }} M_{\text {incd }}\left(1-\frac{M_{\text {incd }}}{K_{\text {incd }}}\right) \\
& \ell<r^{*}: \quad \frac{\mathrm{d} M_{\mathrm{dd}}}{\mathrm{d} \ell}=0 \\
& 1>t^{*}: \quad \frac{\mathrm{d} M_{\mathrm{ed}}}{\mathrm{d} r}=\operatorname{rod}_{\mathrm{od}} M_{\mathrm{inod}}\left(\frac{E_{\mathrm{min}}+M_{\mathrm{ed}}}{K_{\mathrm{ed}}}\right)\left(1-\frac{M_{\mathrm{ed}}}{K_{\mathrm{ad}}}\right)
\end{aligned}
$$
The parameters $t$ and $r^{*}$ are in units of time, $r_{\text {ined }}$ and $r_{o d}$ are in units of time $e^{-1}$, and all other parameters $\left(M_{\text {cd }}, M_{\text {incd. }}\right.$ $\left.K_{\mathrm{cd}}, K_{\mathrm{ined}}, E_{\mathrm{min}}\right)$ are in identical units of either dry mass or

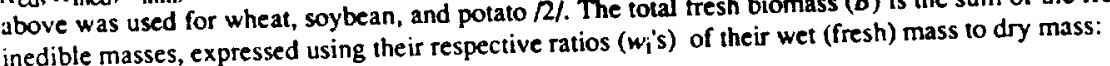

$$
B=w_{\text {ed }} M_{\mathrm{cd}}+w_{\text {ined }} M_{\text {ined }}
$$


We compare the model to data provided by S. Schwartzkopf /4/. He grew wheat at the NASA Ames Research Center under controlled conditions of temperature, humidity, and atmospheric $\mathrm{CO}_{2}(1200 \mathrm{ppm})$. Since we are not concerned here with how growth is affected by changes in these variables (except for humidity, see below), or $\left(B_{60}\right)$, which in his experim la plots this normalized value of $B / B$ maximum total fresh biomass reached during the seed maturation. Figure

Equations (1.2a-b, and 3) are run with $w_{\text {ed }}=1.13 \mathrm{gm}$ fresh per gm dry) $/ 5 /$ and $w_{\text {ined }}=5.7$ (gm fresh per $\mathrm{gm}$ dry) $/ 6 \%$. Other parameters used here are $K_{\mathrm{ed}}=2500, K_{\text {inod }}=3700, E_{\min }=80$, initial $M_{\text {ined }}=10$. In previous models these units have been $\mathrm{gm}$ dry mass $\mathrm{m}^{-2}$, but here the units may be considered arbitrary since to facilitate comparison to Schwartzkopf's data, the model's output is normalized as a natio between total fresh biomass and the total fresh biomass at day 60 . This ratio, $B / B_{60}$, is plotted in figure $1 \mathrm{a}$. Note also that the harvest index, defined as the fraction of the edible dry biomass--here approximately $2500 /(2500+3700)$-is consistent with the value of 0.4 from data $/ 5 /$. The only major unknowns that can influence the shape of the growth curve significantly are the uses $r_{\text {ed }}=r_{\text {ined }}=0.2$ day -1 .

\section{TRANSPIRATION FORMULATION}

Transpiration will probably account for about half the energy balance in the plant growth system of a CELSS. By definition, the total transpiration rate $(\Gamma)$ is proportional to the transpiration rate per unit of inedible biomass $(\gamma)$ and
to the total inedible biomass. Therefore

$$
\Gamma=\gamma M_{\text {ined }}
$$

Following general reasoning such as that given in Gates $\Pi /, \gamma$ is a function of the difference between the partial pressures of water vapor in the leaf $\left(P_{\text {H2O,lesf }}\right)$ and atmosphere $\left(P_{H_{20} \text { sif }}\right)$ and a function of the stomatal resistance $\left(f_{s}\right)$, which itself a complex function of various environmental factors including light, temperature, and $\mathrm{CO}_{2}$.

$$
\gamma=\gamma^{*} f_{\mathbf{b}} f_{\mathbf{a}}
$$

Here we have written $\gamma$ as a product of a humidity factor $\left(\boldsymbol{F}_{\mathrm{h}}\right.$ is a function of $\boldsymbol{P}_{\mathrm{H} 2 \mathrm{O} \text {.lear }}-\boldsymbol{P}_{\mathrm{H} 2 \mathrm{O} \text {,air }}$ ), a stomatal

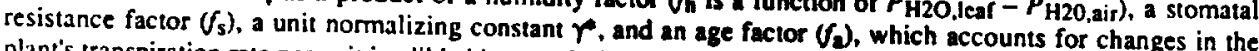
plant's transpiration rate per unit inedible biomass during its life cycle even when all environmental factors $\left(\xi_{\mathrm{h}}, f_{s}\right)$ are constant. A $\gamma=$ constant $=2.4 \mathrm{gm} \mathrm{H} \mathrm{H}_{2} 0$ per $\mathrm{gm}$ dry inedible biomass per day (this gives a rough average of typical wheat under controlled environments $/ 8 /$ ) was used by Rummel and Volk $/ 1 /$, but could not be iested against data during the plani's life cycle at that time. S. Schwartzkopt has been able to take detailed uranspiration data from wheat $/ 4 /$. To facilitate comparison between model and datz the cumulated transpired water at time $t\left(0^{\top} \Gamma \mathrm{d} d\right)$ is

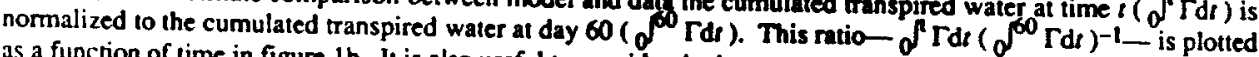
fro function of time in figure $1 \mathrm{~b}$. It is also useful to consider the instantaneous transpiration rate $\Gamma$ (here calculated from the data on cumulated water for any point using the previous and subsequent points), also normalized to the cumulated transpired water at day 60 . This ratio- $\Gamma\left(0^{\infty 0} \Gamma \mathrm{d} t\right)^{-1}$ - has the units of day ${ }^{-1}$, and should be read as the fraction of the total transpired water during the life cycle transpired during a given day; this ratio is plotted in
figure lc.

$$
\begin{aligned}
& \text { Normalized cumulated transpiration }=\frac{\int^{t} \Gamma d t}{\int^{\delta 0} \Gamma d t} \\
& \text { Normalized transpiration rate }=\frac{\Gamma}{\int^{60} \Gamma d t}
\end{aligned}
$$

Schwartzkopfs humidity controls kept the relative humidity $(\mathrm{h}) \boldsymbol{\mu} 0.35$ at the beginning of the experiment, but only maintained th near 0.45 at the end $/ 4 /$; the change was gradual and approximately linear. Assuming leaf $\mathrm{h}=$ 1.0 and air th varied from 0.35 to 0.45 , the humidity factor $f_{h}$, expressed in terms of $t h$ and non-dimensionalized to the final condition, is taken here to have varied linearly from 1.18 at the beginning of the experiment ( $(1$ $0.35) /(1-0.45))$ to 1.00 at the end. Furthermore, we set $\gamma^{*}=2.4 \mathrm{gm} \mathrm{H} \mathrm{H}_{2} \mathrm{O}$ per gm dry inedible biomass per day, and since environmental conditions were approximately constant, $f_{3}=1.0$ for the duration of the experiment. We test several cases of the age factor $f_{2}$.

The first case has $f_{\mathbf{2}}=1.0=$ constant (or, $\alpha=0$, see below). Output from the model for cumulated transpiration and iranspiration rate is nomalized to the cumulated transpiration at day 60 using equations $6 a-b$, like the experimental data. Note this normalization effectively eliminates dependence of the results on $\gamma^{*}$. Results with $f_{a}$ 
constant are plotted against data in figures $1 \mathrm{~b}$ and $1 \mathrm{c}$. Although the general shape of the cumulated transpiration data is matched by the model (see figure $\mid \mathrm{b}$ ), the empirical value is significantly underestimated during the middle one-third of the life cycle. The underestimation is even clearer in the rate results, shown in figure lc. For the first one-half of the life cycle, the transpiration rate in the model is much 100 low.

A second case explores the possibility that the transpiration rate per unit inedible biomass is substantially higher A the plant is younger than when mature. A convenient way of parameterizing this process that takes into account the apparent steadiness of the transpiration rate during the second one-half of the life cycle, when the itself is relatively miximum and constant, is to write $f_{a}$ as a function of $M_{\text {incd: }}$

$$
f_{a}=1+\alpha\left(1-\frac{M_{\text {incd }}}{K_{\text {incd }}}\right)
$$

Here the term $\alpha$ is an enhancement of transpiration rate per unit biomass when the plant is young. Note $f_{a 1}=1.0$ when $M_{\text {ined }}=K_{\text {incd }}$. The model output for cumulated transpiration and transpiration rate for this second modelwhich uses $\alpha=1$, rather than $\alpha=0$-is shown in figures $1 b$ and lc. Overall better fit to the data is apparent, in

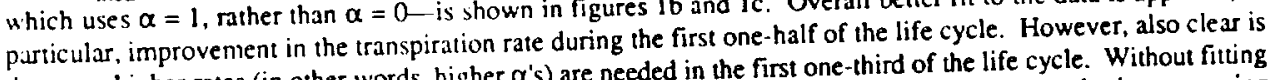
that even higher rates (in other words, higher $\alpha$ 's) are needed in the first one-thres of the the transpiration to varying the data even further, we have nevertheless demonstrated the possibili

\section{CONCLUSION}

Models such as these are the best way of examining the "interactions of assumptions" $/ 9 /$. Considering the overal] (he logistic growth equations combined with the assumption that the rate of transpiration per unit inedible mass decreases during the life cycle of the crop will generally reproduce the data and will probably be adequate in

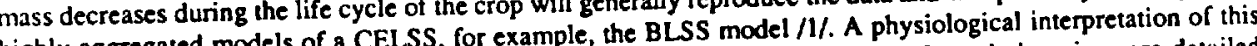
highly aggregated models of a transpiration formulation and comparison or these findicte to a CELSS model) will

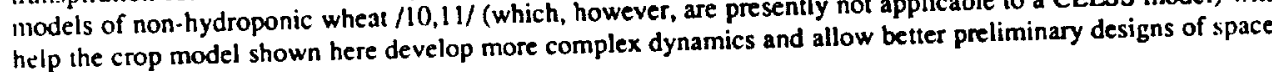
agricultural systems.

\section{ACKNOWLEDGEMENTS}

Research time for $T$. V. was partially provided by NASA-Ames Joint Research Interchange NCA2-101. We thank $\mathrm{T}$. Tibbitts for a thoughtful and important review.

\section{REFERENCES}

1. J. D. Rummel and T. Volk, A Modular BLSS Simulation Model, Adv. Space Res., 7, \# 4, 59-67 (1987).

2. T. Volk, Modeling the growth dynamics of four candidate crops for controlled ecological life support systems. Final repor to NASA/ASEE Summer Faculty Fellowship Program at Johnson Space Center, NGT44-001-800, 33-1 to 33-17 (1987).

3. T. Volk and $H$. Cullingford, Crop growth and associated life support for a lunar farm, Lunar Bases and Space Activities of the 21 st Century, in press (1989).

4. S. Schwartzkopf, private communication (1987).

5. B. G. Bugbee and F. B. Salisbury, Studies on maximum yield of wheat for the controlled environments of B. Bulorical Life Support Systems: CELSS '85 Workshop, eds. R. D. MacElroy, N. V. Manello, and D. T. Smernoff, NASA-TM-88215, p. $447-486$ (1986).

6. B. G. Bugbee, private communication (1988).

7. D. M. Gates, Biophysical Ecology, Springer-Verlag, New York (1980).

8. B. G. Bugbee, private communication (1986).

9. J. F. Reynolds and B. Acock, Modeling approaches for evaluating vegetation responses to carbon dioxide concentration, eds. B. R. Strain and J. D. Cure, Direct Effects of Increasing Carbon Dioxide on Vegetation. DOE/ER-0238. U. S. Dept. of Energy, Wash., D. C., p. 33-51 (1985). 
10. T. Hodges and E. T. Kanemasu. Modeling daily dry matter production of winter wheat, Agronomy Journal.
$69,974.978$ (1977). 11. S. J. Maas and G. F. Arkin, TAMW: A wheat growth and development simulation model, (Research Center
Program and Model Documentation. No. 78-1), Blackland Research Center at Temple, Texas Agriculture
Experiment Station, College Station, Texas (1980).
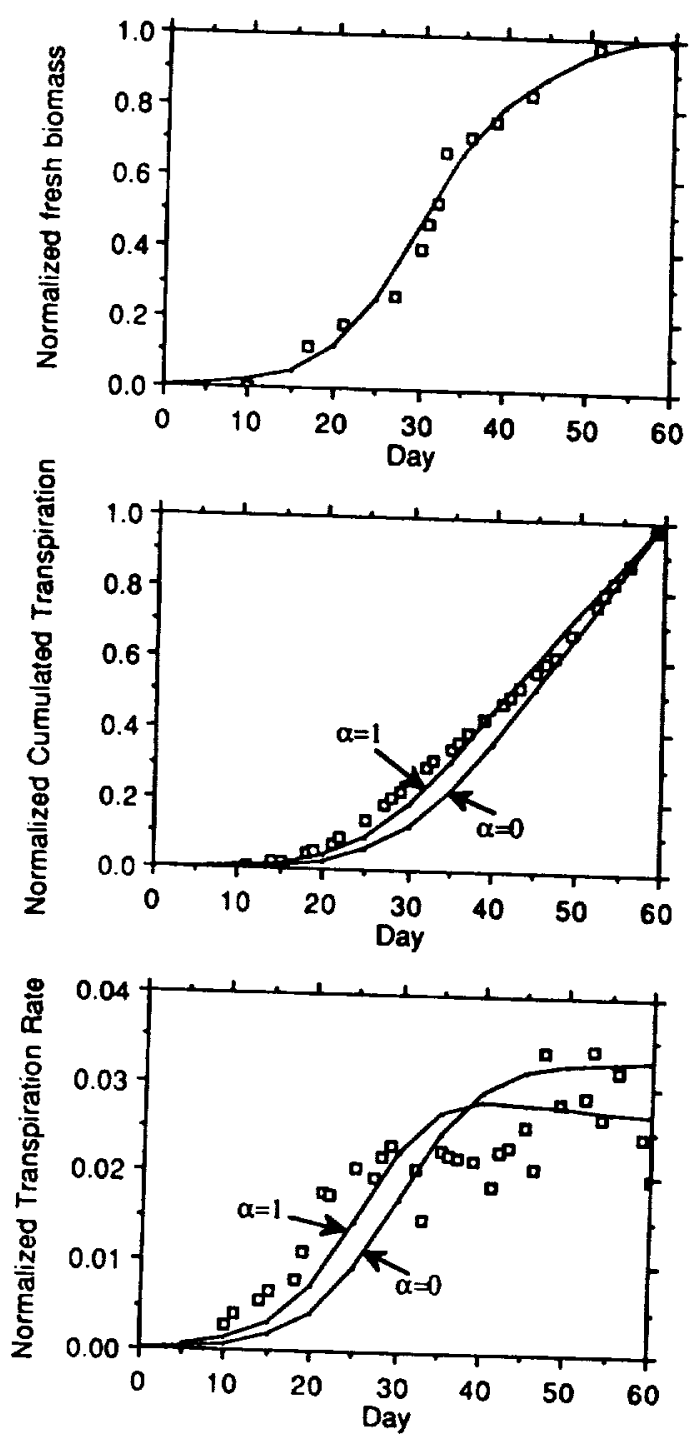

Fig. 1. Model results (lines) and empirical data (points) of (a, top) fresh biomass, (b, middle) Results in (b) and (c) are shown for transpiration rate, all nomalized as described in the text. factor $f_{\mathrm{a}}$ in the transpiration rate. When $\alpha=0$ alues of $\alpha ; \alpha$ controls the time-dependence of the age linearly from about 2 in early growth to about 1 in $f_{a}$ is constant; when $\alpha=1, f_{a}$ decreases nondo not vary as a function of $\alpha$. See equat 1 in late growth (see text). Results for biomass in (a) transpiration and nomalized transpiration mate. $6 a-b$ for definition of the nomalized cumulated 